\title{
GBParsy: A GenBank flatfile parser library with high speed Tae-Ho Lee ${ }^{1,2}$, Yeon-Ki Kim*2 and Baek Hie Nahm*1,2
}

Address: ${ }^{1}$ Division of Bioscience and Bioinformatics, MyongJi University, Yongin, Kyonggido, Republic of Korea and ${ }^{2} \mathrm{Genomics}$ Genetics Institute, GreenGene BioTech Inc., Yongin, Kyonggido, Republic of Korea

Email: Tae-Ho Lee - thlee@bio.mju.ac.kr; Yeon-Ki Kim* - kim750a11@gmail.com; Baek Hie Nahm* - bhnahm@mju.ac.kr

* Corresponding authors

Published: 25 July 2008

BMC Bioinformatics 2008, 9:321 doi:10.1|86/|47|-2/05-9-32|

This article is available from: http://www.biomedcentral.com/I47I-2105/9/32I

(c) 2008 Lee et al; licensee BioMed Central Ltd.

This is an Open Access article distributed under the terms of the Creative Commons Attribution License (http://creativecommons.org/licenses/by/2.0), which permits unrestricted use, distribution, and reproduction in any medium, provided the original work is properly cited.

\begin{abstract}
Background: GenBank flatfile (GBF) format is one of the most popular sequence file formats because of its detailed sequence features and ease of readability. To use the data in the file by a computer, a parsing process is required and is performed according to a given grammar for the sequence and the description in a GBF. Currently, several parser libraries for the GBF have been developed. However, with the accumulation of DNA sequence information from eukaryotic chromosomes, parsing a eukaryotic genome sequence with these libraries inevitably takes a long time, due to the large GBF file and its correspondingly large genomic nucleotide sequence and related feature information. Thus, there is significant need to develop a parsing program with high speed and efficient use of system memory.

Results: We developed a library, GBParsy, which was $C$ language-based and parses GBF files. The parsing speed was maximized by using content-specified functions in place of regular expressions that are flexible but slow. In addition, we optimized an algorithm related to memory usage so that it also increased parsing performance and efficiency of memory usage. GBParsy is at least $5-100 x$ faster than current parsers in benchmark tests.

Conclusion: GBParsy is estimated to extract annotated information from almost $100 \mathrm{Mb}$ of a GenBank flatfile for chromosomal sequence information within a second. Thus, it should be used for a variety of applications such as on-time visualization of a genome at a web site.
\end{abstract}

\section{Background}

Large volumes of information have been rapidly accumulating since the shotgun DNA sequencing technology was introduced $[1,2]$. Currently, GenBank volume size is rapidly increasing, with more than 370 complete microbial genomes and over 104 assemblies of eukaryote genomes deposited over the year 2006 alone [3]. This has led to the question of how this invaluable information can be dealt with by computer programs. One of the primary concerns is how to efficiently convey huge volumes of sequence data and their annotated information to researchers. For these reasons, many sequence formats such as Abstract Syntax Notation One (ASN.1), Extensible Markup Language (XML) and GenBank flatfile (GBF) format have been used to manage sequences for distinct purposes and usages. Among these, the ASN.1 and XML format files are generally known to be difficult for a user to directly get information since the formats are designed to specify complex data structures in a machine and programming language. Thus, they are used for storing and exchanging data between computer systems. In contrast, GBF format has become one of the most popular because of its 
detailed sequence features and ease of readability and accessibility unlike machine-friendly ASN.1 or XML format. The format has been widely adopted to describe not only a relatively short individual gene sequence, but also long sequences, such as eukaryotic genome sequences in animals and plants $[4,5]$. To use the data in the file by a computer, a parsing process is required and is performed according to a given grammar for the sequence and the description in a GBF. Therefore, the GBF parser has become a routine program in bioinformatics. NCBI C/ $\mathrm{C}++$ toolkit [6] provides a lot of functions to deal with a sequence including parsers for ASN.1 format since NCBI employed the format as a central data format, but the toolkit does not provide a parser for the GBF. Thus, currently, several parser libraries for the GBF have been developed by other groups, such as BioPython [7], BioPerl [8] and the AJAX library in the EMBOSS package [9]. However, parsing a large GBF file (such as a eukaryotic genome sequence) with these libraries inevitably takes a long time since the parser libraries were not designed just for parsing speed. For example, parsing time for the GBF of a chromosomal sequence of Arabidopsis thaliana by the GBF parser of BioPython is estimated to be 109.2 seconds (Table 1). Thus, the biological community needs a faster parser that can parse a large GBF file, such as a eukaryotic chromosome, in a single-digit number of seconds at the personal computer level. We developed the GBParsy library [see Additional file 1], a C language-based parser with improved speed and efficient use of memory. In addition, we developed GBParsyPy, a GBParsy wrapper for the Python programming language.

\section{Implementation}

In the design of the GBParsy library, we focused on the speed and the efficient use of system memory. At first, we improved parsing speed by using customized parsing functions in place of the regular expression functions. Although regular expressions are frequently used to parse

Table I: Comparative performance of GBParsy and other publicly available GBF parsers

\begin{tabular}{|c|c|c|}
\hline Source & A. thaliana & M. musculus \\
\hline Accession & NC 003070 & NT 039207 \\
\hline Sequence Length (Mbp) & 30.4 & 116.4 \\
\hline File Size (MB) & 59.0 & 144.0 \\
\hline GBParsy & $0.9 \pm 0.1(1.0)$ & $2.4 \pm 0.3(1.0)$ \\
\hline GBParsyPy & $1.7 \pm 0.1(1.8)$ & $3.0 \pm 0.5(1.2)$ \\
\hline EMBOSS & $9.7 \pm 0.2(10.7)$ & $12.7 \pm 0.3(5.3)$ \\
\hline BioPerl & $45.7 \pm 0.4(50.3)$ & $38.7 \pm 0.3(16.0)$ \\
\hline BioPython & $109.2 \pm 0.4(120.3)$ & $39.7 \pm 0.2(16.4)$ \\
\hline
\end{tabular}

Elapsed time (sec) taken by each program was measured in 50 separate runs; mean \pm standard deviation (SD) for these runs are shown. Average fold slowness in parenthesis is the ratio of each observed mean time to the one taken by the GBParsy test program. strings because of their flexibility, they lower the speed of a program as a trade-off for their flexibility. Further enhancement was achieved by optimizing an algorithm related to memory usage. We tried to reduce the number of functions in the GBParsy that were for allocating new memory space and for moving data between the memory spaces, which are both time-consuming processes; thus, we were able to save system memory as well as increase performance.

The functions in GBParsy are categorized into three groups. The functions in the common group perform general operations, such as handling white spaces or lines. Each field, such as features or sequences in GBF, is handled by distinct functions included in the parsing group. The functions included in the common and parsing groups are only used internally. The functions in a user group are directly used by the user, and are implemented to handle a GBF file or parsed data. For example, the user can get a parsing result of a GBF file by calling the 'parseGBFF' function with the file name.

Whole entry fields of a GBF file are parsed by calling the 'parseGBFF' function with the file name (Figure 1), and the parsing results are stored in 5 types of structures (Figure 2), which is a collection of variables and a kind of data type in C language. Gb_data structure is a main structure and a gb_data structure contains one GBF data. Each record in the reference entry and in the feature table of the GBF data is stored gb_reference and gb_feature structure, respectively. Gb_location and gb_qualifier, which is a substructure of gb_feature, contain information of a location and qualifiers, respectively. In the case of GBParsyPy, while the parser uses proper data types of the Python programming language (such as dictionary and tuple type in place of structure type of the $\mathrm{C}$ language), the formation of a parsing result by GBParsyPy is generally similar to that by GBParsy. For example, the main structure of GBParsy, gb_data, is implemented as a dictionary where each element in the dictionary is matched to each variable in the main structure.

\section{Results \& Discussion Benchmarking test}

The two largest GBF files of A. thaliana chromosome 1 ( $\underline{\text { NC 003070) }}$ and Mus musculus chromosome 2 (NT 039207) were downloaded from GenBank ftp:// ftp.ncbi.nih.gov/genomes/for the performance test. The performance of GBParsy was ascertained by benchmark testing with an Athlon 64 Processor 3200+ system with 2 GB main memory in Linux for the two publicly available chromosome sequences. To test the performance, we used an incorporated program in the EMBOSS (ver. 5.0.0) package, 'extractfeat', which parses GBF with the AJAX library and extracts specific sequences from the file. Also, 
(6)

\begin{tabular}{|c|c|}
\hline & \\
\hline LOCUS & (2) $116366104 \mathrm{bp}(3)$ DNA \\
\hline SFINITIO(7) & Mus musculus chromosome 2 genomic contiq, strain c57BL/6 \\
\hline CESSION ( 8 ) & 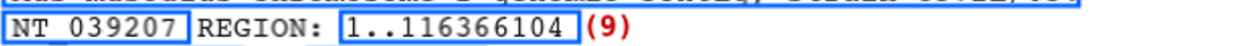 \\
\hline VERSION $(10)$ & 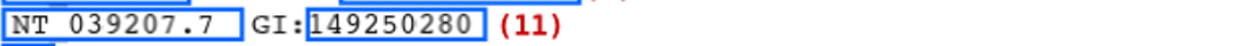 \\
\hline KEYWORDS(12) &. \\
\hline SOURCE (13) & Mus musculus (house mouse) \\
\hline ORGANI(14) & Mus musculus \\
\hline & $\begin{array}{l}\text { Eukaryota; Metazoa; Chordata; Craniata; Vertebrata; Euteleostomi; } \\
\text { Mammalia; Eutheria; Euarchontoglires; Glires; Rodentia; } \\
\text { Sciurognathi; Muroidea; Muridae; Murinae; Mus. }\end{array}$ \\
\hline BFERENC( 16) & 1 ( \\
\hline AUTHORS & $\begin{array}{l}\text { Griffiths-Jones S, Grocock RJ, van Dongen S, Bateman A and Enright } \\
\text { AJ. }\end{array}$ \\
\hline TITLE $(18$ & miRBase: microRNA sequences \\
\hline JOURNA(19) & \begin{tabular}{|lllll} 
Nucleic Acids Res. 34 (DATABASE ISSUE), D140-D144 (2006) \\
\end{tabular} \\
\hline & 16381832 \\
\hline DMMENT & $\begin{array}{l}\text { GENOME ANNOTATION REFSEQ: Features on this sequence have been } \\
\text { produced for build } 37 \text { version } 1 \text { of the NCBI's genome annotation } \\
\text { [ see documentation]. } \\
\text { On or before Jun } 20,2007 \text { this sequence version replaced } \\
\text { gi: } 94367675, \text { gi: } 94367450 \text {. } \\
\text { The DNA sequence is part of the largely finished reference sequence } \\
\text { (C57BL/6J) that contains small amounts of WGS and HTGS Draft } \\
\text { sequence. This assembly was performed by NCBI in consultation with } \\
\text { the Mouse Genome Sequencing Consortium. } \\
\text { Name: Mm2 } 3924737 \text {. }\end{array}$ \\
\hline
\end{tabular}

FEATURES Location/Qualifiers

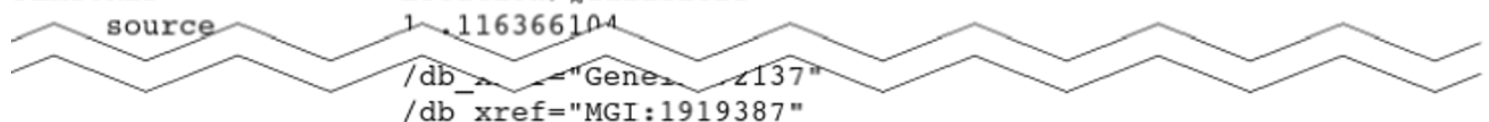

(22) CDS

(23) complement ( j (24) 732163. .732320, (25) )69..738109,

$740920 \ldots 741096,741979 \ldots 742085,742176 \ldots 742216$,

$743661 \ldots 743694,752575 \ldots 752668,753598 \ldots 753687$,

$756026 \ldots 756210,757489 \ldots 757886))$

(27) gene- "Wdsub1" (28) (26)

/GO_component="cellular_component; ubiquitin ligase

complex"

/GO_function="molecular_function; ubiquitin-protein ligase

activity"

/GO_process="biological_process; protein ubiquitination"

/note="novel protein containing WD40, SAM and U-box

domains"

/ codon_start $=1$

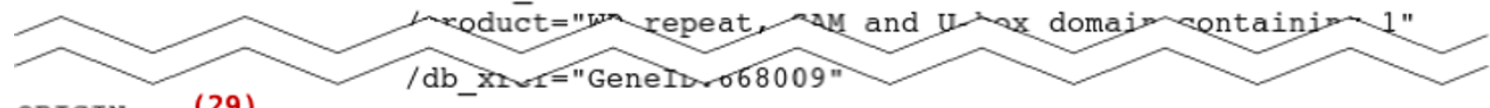

ORIGIN (29)

1 ataaaccac cattcattga cttagtactg tcattgaatt cactatcagt tctaccatat

61 actttgttgt tattcttgag acttagagt ttctacgta tatcatatgg atcagagttg

121 caaggttct tctgagaaat ccagtggtag cccagtggaa tttgtggaaa gtgtgactgg

181 ctttcttt tgccctcaaa aattgcctga atttaacaa agtataacat gtctctgtgt

Figure I

Example of a parsing result. Each box represents a datum as a result of parsing the GBF file, NT 039207. A number in parenthesis is denoted as such in Figure 2. 


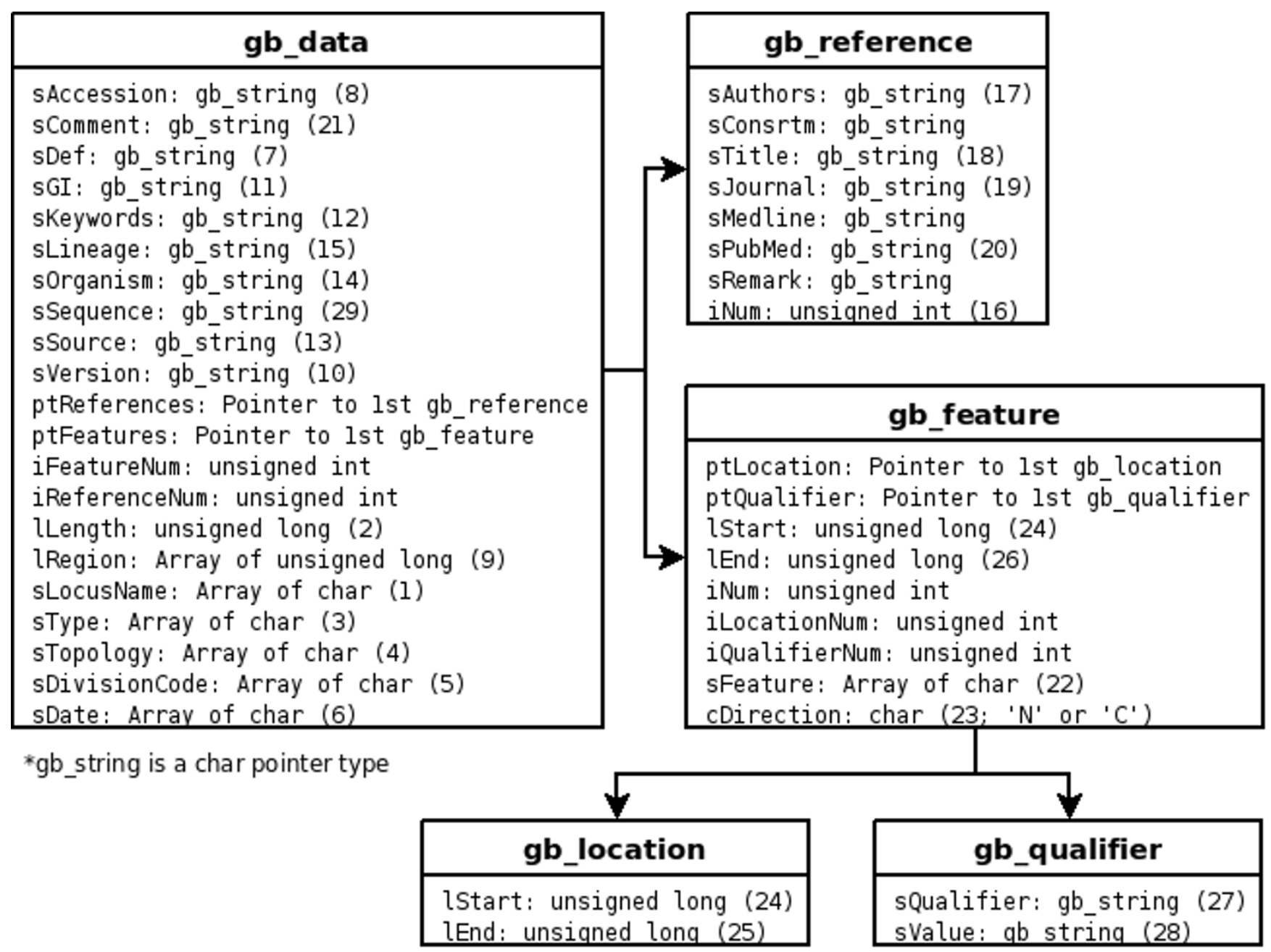

Figure 2

Structures of a parsing result of GBParsy. The parsing result of GBParsy is organized with five structures that are represented by a box. A large bold title such as gb_data represents the name of the structure and items in a box represent elements of the structure. A variable name and its data type in $C$ language are denoted on the left- and the right-side of the colon, respectively. A number in parenthesis links with the number in Figure I and it represents a datum block stored in the variable.

we developed testing programs generating similar output with the 'extractfeat', for GBParsy, GBParsyPy, BioPerl (ver. 1.4) and BioPython (ver. 1.43), respectively. To compare the performance of each library, we extracted whole tRNA sequences from a GBF file with each testing program and determined the elapsed time. The average and standard deviation of the elapsed times (sec) for each program were measured in 50 separate runs. Slower folds for each parser were calculated against that of GBParsy to represent the performances.

\section{GenBank flatfile parser}

Elapsed times were measured for each program in parsing two GBF files containing large chromosomal sequences and annotation of the A. thaliana chromosome (NC 003070) and M. musculus chromosome
(NT 039207), (Table 1), which are known to be the largest files with proper annotation in plants and animals, respectively. When GBParsy was used, it took $0.9 \mathrm{sec}$ and $2.4 \mathrm{sec}$ to parse the chromosomal sequences from Arabidopsis and mouse, respectively. For the chromosomal sequences of Arabidopsis, the average fold slowness indicates that it was two orders faster than the parser of BioPython and more than 10 times faster than the AJAX parser of the EMBOSS package. In the case of the mouse chromosome sequence information, GBParsy was 16 times faster than the parser of BioPython and was five times faster than the AJAX library of the EMBOSS package. The difference in parsing performance, between the two GBF files, by GBParsy and the others resulted from percent sequence portion and/or the likewise annotation portion in the files. Since the structure of the sequence region is simpler 
than the annotation region of the feature fields, the difference in parsing efficiency between parsers while parsing a sequence region is small. The sequence portion of chromosomal information in Arabidopsis is about half of the file, while that in the mouse is over $80 \%$. Therefore, GBParsy is more effective for a GBF file containing a large volume of annotated information than a file with poor annotation.

When the main memory is not sufficient, a computer system generally uses virtual memory, which is substantially slower than main memory. Thus, a program requiring a large volume of memory needs a long time for its execution on a system such as a personal computer. Accordingly, we tested whether the more limited use of memory by GBParsy influenced the speed of the program. This can also be estimated by measuring elapsed times. For example, when we parsed the chromosomal sequence file from mouse using a system with $512 \mathrm{MB}$ main memory, the AJAX library consumed 150 seconds while GBParsy processed in eight seconds. The result reflects that GBParsy efficiently uses system memory.

GBParsyPy is a GBParsy for Python, which is a representative scripting language. Because GBParsyPy adopted GBParsy as a core parser, it inherited all of its features from GBParsy, performing at almost paralleled speed and with efficient use of memory. Thus, in the case of the $M$. musculus chromosome, GBParsyPy was over 10 times faster than the parser of BioPython or that of BioPerl. Although we did not develop a Perl version, GBParsy could be easily adopted by the language. To test usability of GBParsyPy in an application program, we developed a new program, ChrDiagram http://gbfp.googlecode.com/ files/chrdiagram-0.1.1.tgz which parses a GBF file with a parser either GBParsyPy or BioPython and draws a sequence diagram from the parsed result with GenomeDiagram [10]. GBParsyPy just took about 7 seconds in parsing the five Arabidopsis chromosome sequences whereas BioPython took over 5 minutes, when we drew genome diagrams of the chromosomes [see Additional file 2] with ChrDiagram at the benchmarking test system. Consequently, to draw the chromosome diagrams with full-features it took about 1 minute with GBParsyPy whereas it took over 6 minutes with BioPython. These results show that usability of application programs can be enhanced with a high speed parser.

\section{Conclusion}

As more rapid genome sequencing technologies emerge, and more information is accumulated, the size of the GBF flatfile will be increased. As exemplified for parsing GBF files of eukaryotic chromosomes, GBParsy and GBParsyPy would be expected to significantly improve the parsing speed and the efficient use of system memory. Thus, our program would be useful for various applications that are difficult or impossible with a slow parser, such as on-time visualization of a chromosome sequence in an annotation program or web-based server.

\section{Availability and requirements}

Project name: GBParsy (A GenBank flatfile parser library with high speed)

Project home page: http://code.google.com/p/gbfp/

Operating system(s): any OS that supports GCC environment

Programming language: GCC

Other requirements: Python

License: GNU GPL

Any restrictions to use by non-academics: None

\section{Authors' contributions}

T-HL developed the parser and drafted the manuscript. Y-KK provided substantial advice and revised the manuscript. BHN provided substantial advice and guidance during all phases of the project and assisted in the drafting of the manuscript. All authors read and approved the final manuscript.

\section{Additional material}

\section{Additional file 1}

GBParsy (and GBParsyPy) version 0.60. This compressed file contains GBParsy and GBParsyPy source codes and additional files such as example programs and instruction for installation. The latest version is available at GBParsy homepage http://code.google.com/p/gbfp/.

Click here for file

[http://www.biomedcentral.com/content/supplementary/14712105-9-321-S1.tgz]

\section{Additional file 2}

Diagram of Arabidopsis chromosome 1-5. The diagrams were drawn by ChrDiagram, in which sequence data were parsed by GBParsyPy and handled with GenomeDiagram. An orchid bar, orange red bar and olive bar on the 1st, 2nd and 3rd track denote a gene, a tRNA and a miscellaneous feature such as transposable element, respectively.

Click here for file

[http://www.biomedcentral.com/content/supplementary/14712105-9-321-S2.zip]

\section{Acknowledgements}

This work was supported by the Crop Functional Genomics Center of the 2 I st Century Frontier Research Program, funded by the Ministry of Science and Technology (grant to B.H. Nahm) and by the BioGreen2I Program 
(grants to Y.-K. Kim and B.H. Nahm), RDA of the Republic of Korea. T.-H. Lee and B.H. Nahm were supported by BK2I. We thank the staff of the GCC and Python programs for granting access to the open source software, and Dr. Jonathan Kans at National Center for Biotechnology Information (NCBI) for helpful advice.

\section{References}

I. Sanger F, Nicklen S, Coulson AR: DNA sequencing with chainterminating inhibitors. Proc Natl Acad Sci USA 1977, 74:5463-5467.

2. Adams MD, Celniker SE, Holt RA, Evans CA, Gocayne JD, Amanatides PG, Scherer SE, Li PW, Hoskins RA, Galle RF, George RA, Lewis SE, Richards S, Ashburner M, Henderson SN, Sutton GG, Wortman IR, Yandell MD, Zhang Q, Chen LX, Brandon RC, Rogers YH, Blazej RG, Champe M, Pfeiffer BD, Wan KH, Doyle C, Baxter EG, Helt G, Nelson CR: The genome sequence of Drosophila melanogaster. Science 2000, 287:2185-2195.

3. Benson DA, Karsch-Mizrachi I, Lipman DJ, Ostell J, Wheeler DL: GenBank. Nucleic Acids Res 2007, 35:D21-D25.

4. Waterston RH, Lindblad-Toh K, Birney E, Rogers J, Abril JF, Agarwal $P$, Agarwala R, Ainscough R, Alexandersson M, An P, Antonarakis SE, Attwood J, Baertsch R, Bailey J, Barlow K, Beck S, Berry E, Birren B, Bloom T, Bork P, Botcherby M, Bray N, Brent MR, Brown DG, Brown SD, Bult C, Burton J, Butler J, Campbell RD, Carninci P: Initial sequencing and comparative analysis of the mouse genome. Nature 2002, 420:520-562.

5. Theologis A, Ecker JR, Palm CJ, Federspiel NA, Kaul S, White O, Alonso J, Altafi H, Araujo R, Bowman CL, Brooks SY, Buehler E, Chan A, Chao Q, Chen H, Cheuk RF, Chin CW, Chung MK, Conn L, Conway AB, Conway AR, Creasy TH, Dewar K, Dunn P, Etgu P, Feldblyum TV, Feng J, Fong B, Fujii CY, Gill JE: Sequence and analysis of chromosome I of the plant Arabidopsis thaliana. Nature 2000, 408:816-820.

6. NCBI C/C++ Toolkit [http://www.ncbi.nlm.nih.gov/books/ bv.fcgi?rid=toolkit.TOC]

7. BioPython [http://www.biopython.org/]

8. Stajich JE, Block D, Boulez K, Brenner SE, Chervitz SA, Dagdigian C, Fuellen G, Gilbert JG, Korf I, Lapp H, Lehvaslaiho H, Matsalla C, Mungall CJ, Osborne BI, Pocock MR, Schattner P, Senger M, Stein LD, Stupka E, Wilkinson MD, Birney E: The Bioperl toolkit: Perl modules for the life sciences. Genome Res 2002, | 2:161|-|6|8.

9. Rice P, Longden I, Bleasby A: EMBOSS: the Euro-pean Molecular Biology Open Software Suite. Trends Genet 2000, 16:276-277.

10. Pritchard L, White JA, Birch PR, Toth IK: GenomeDiagram: a python package for the visualization of large-scale genomic data. Bioinformatics 2006, 22:616-617.
Publish with Bio Med Central and every scientist can read your work free of charge

"BioMed Central will be the most significant development for disseminating the results of biomedical research in our lifetime. "

Sir Paul Nurse, Cancer Research UK

Your research papers will be:

- available free of charge to the entire biomedical community

- peer reviewed and published immediately upon acceptance

- cited in PubMed and archived on PubMed Central

- yours - you keep the copyright
BioMedcentral 